\title{
Boosting the Performance of Quantum Repeaters
}

\author{
Multiplexing techniques could boost the chances of achieving end-to-end \\ entanglement of a signal in a trapped-ion-based quantum-computer \\ network. \\ By Erika K. Carlson
}

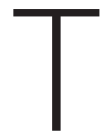

o create a large-scale network of quantum computers, researchers need to develop devices that can amplify and reinforce the signals that these networks will carry (see Research News: The Key Device Needed for a Quantum Internet). These devices, known as quantum repeaters, play an important role in distributing entanglement over the networks. Now, Prajit Dhara of the University of Arizona and colleagues have investigated how multiplexing techniques impact the rates of end-to-end entanglement in a network of quantum repeaters made of trapped ions [1]. The researchers say that their investigation helps pave the way for implementing trapped-ion quantum repeaters in quantum networks.

The quantum-repeater-network design that Dhara and colleagues considered is based on repeaters made of trapped-ion qubits, one of the front-running qubit types for quantum computers. The network of repeaters incorporates both spatial and temporal multiplexing-techniques for

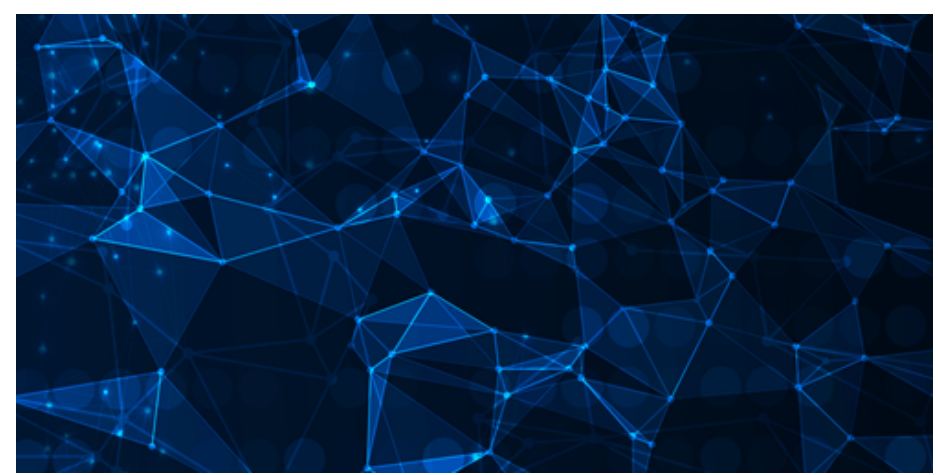

Credit: krass99/stock.adobe.com transmitting multiple, simultaneous signals within a communication channel. In spatial multiplexing, each pair of adjacent repeaters makes multiple, parallel attempts to entangle with one another to improve the chances of achieving entanglement across the network. In temporal multiplexing, the network as a whole iterates the entanglement attempts, balancing the benefit of multiple attempts with the downside of longer wait times.

Dhara and colleagues find that the network design that they considered has increased rates of entanglement compared to those predicted in previous studies. The team also add in predictions for the ion resources that multiplexed quantum repeaters need in order to boost entanglement rates, calculating the best entanglement rates achievable for a given set of resources.

Erika K. Carlson is a Corresponding Editor for Physics based in New York City.

\section{REFERENCES}

1. P. Dhara et al., "Multiplexed quantum repeaters based on dual-species trapped-ion systems," Phys. Rev. A 105, 022623 (2022). 